\title{
Static realization of reliable positional indication
}

\author{
A.V. Bushma, G.A. Sukach \\ Institute of Semiconductor Physics, NAS of Ukraine, 45 Prospect Nauky, 03028 Kyiv, Ukraine \\ Phone: +380 (44) 265 6188; fax: +380 (44) 265 5430; e-mail: bushma@isp.kiev.ua
}

\begin{abstract}
The highly reliable position information model for optoelectronic display systems is offered. Logical operators that describe static formation of this model are obtained and analyzed. The main features of alphabets for two variants of this information model and information fields for their synthesis are investigated. Also offered are mathematical models for specific devices intended for highly reliable positional imaging information at the display consisting of elements with one common electrode. Analized and represented is their circuit solution.
\end{abstract}

Keywords: positional indication, reliability, modeling, display, LED, bar graph array, optoelectronics, logic element, digital structure, integrated circuit.

Paper received 31.01.02; revised manuscript received 15.02.02; accepted for publication 05.03.02.

\section{Introduction}

One of the main parameters of modern informationmeasuring systems is high reliability of data transfer to the operator. This reliability is determined by two major factors: first, by a configuration and qualitative parameters of apparatus means, and, second, by specific features of information transfer from an imaging system to the operator [1,2].

Estimation of the first component is traditional and can be carried out using calculation of a probability of the device fail-proof operation within a definite time interval obeying some analytical or experimental methods $[3,4]$. Taking the second method into consideration is more complicated and based on man-resourse-loading experimental methods that envisage using a considerable number of expert conclusions made by several operator groups for validity of results obtained. In the course of the experiment, created are data massifs when reading information out in different conditions [5, 6]. Practically, in the most cases, more effective are analysis and evaluation of some information model (IM) that can reflect the state of a controlled object [7]. Such approach determines the considerable interest to creation and research of IMs for displays of different types.

In displays, information is transferred to an operator via some system of signs corresponding to imaged values. The set of used symbols forms IM. One of the most reliable discrete realization for such information process is based on scale data representation, which is conditioned by both the high level of adequacy between symbol shapes and meanings and an informational excess of scale indication.

\section{Information models of scale indication}

Two IM are most widely used in devices for measuring and computational techniques in the case of scale data representation. These are bar graph and positional IMs with corresponding specific alphabets for visual symbols. In the first case, indication data is fixed through a position of an optical non-uniformity in the indicator information field, and in the second one it is conditioned by both an length and position of the indication end formed by the optical non-uniformity relatively scale marks. As for scales based on active elements, e.g., LEDs, it is either an illuminating mark or illuminating line on the scale, respectively.

It is obvious that in portable and mobile systems with scales using LEDs, more actual is usage of the positional IM, since this form of data representation provides their high efficiency. Within the framework of this model, the respective level of efficiency and reliability of these devices is reached due to analysis and separation of functional and technical solutions for their units, which is the most successfully solved using informational process modeling in imaging systems [2, 7]. Among determinated mathematical methods, the most widely spread means for analytic description, while data processing in informational systems, are theory-of-sets and logical methods [2, 8].

In this work, we made an analysis of logic regularities that are characteristic for a synthesis of the positional IM at a bar graph display in optoelectronic informational-and-measuring systems. 


\section{A.V. Bushma et al.: Static realization of reliable positional indication}

\section{Operation of the information model}

Any display is designed to transfer operator some information $I$ that can be represented by a set of annunciations

$$
I=\left\{I_{1}, I_{2}, \ldots, I_{i}, \ldots, I_{l-1}, I_{l}\right\}
$$

where $I_{i}$ is $i$-th meaning of annunciations ordered in such manner that $I_{i-1}<I_{i}$, and $i=\overline{1, l}$.

Data from an imaging device are transferred to the operator per IM elements that are visual symbols $S_{v}$. As a rule, each annunciation is corresponded by the unique symbol. The sense of these symbols is common for the whole set $I$, e.g. one deals with a voltage indicator, and the voltage value runs along a series of meanings. Therefore, the set (1) can be represented via respective symbols $S_{v}$ as

$I=\left\{I\left(S_{1}\right), I\left(S_{2}\right), \ldots, I\left(S_{v}\right), \ldots, I\left(S_{q-1}\right), I\left(S_{q}\right)\right\}$

where $I\left(S_{v}\right)$ is the $v$-th meaning that corresponds to the $S_{v}$ symbol, while $v=\overline{1, q}$.

The set used $S_{v}$ symbols forms the IM alphabet $\Omega_{\mathrm{IM}}$. Its length equal to the $s$ number of various symbols in this IM is determined by a final set of allowed states in the display information area. This area consists of $\alpha_{i}$ elements that form the set $A$ and can be described as $A=\left\{a_{1}, a_{2}, \ldots, a_{i}, \ldots, a_{p-1}, a_{p}\right\}$

where $p$ is the total number of information area elements (IAE).

From the viewpoint of electrical circuits, IAEs are most often two-terminals, and their mutual connections have a linear (one-coordinate) or matrix (two-coordinate) arrangement. In this approach, a spatial location of elements is determined by the topology of used IM and is invariant relatively to electric connections.

It is obvious that a visual form of the scale information representation assumes the presence of a weighting factor intrinsic to each display element. As a rule, any meaning of the weighting factor is linked with a spatial location of IAE on the display information area and is proportional to its number $i$ in the scale. Reading information out is realized in accord with the weighting factor meaning relatively to scale marks, while the scale serving as a multi-channel measure [1].

Image of symbols is formed in the display from discrete elements $\alpha_{i}$ in accordance with a given IM. A control circuit creates necessary optical non-uniformity using excitation of optoelectronic elements $\alpha_{i}$ per respective electric signals. To reach it, formed is the set $G(v)$ of $\alpha_{i}$ elements, composition of which is determined by the number $v$ of the visual symbol $S_{v}$.

In the case, the set $G(v)$ is a sub-set of the set $A$, oneto-one correspondence between the synthesized symbol $S_{v}$ and the set $G(v)$ being functionally provided. It is the main condition to adequately represent the meaning of a measured value and data authentically read by an operator. This representation of data on a bar graph array for the IM with a continuous visual pattern can be written using the following unification operator

$$
S_{v} \Leftrightarrow G(v)=\bigcup_{i=i_{1}}^{i_{2}} a_{i}
$$

where $i_{2}, i_{1}$ are positional numbers of the initial and final elements in the formed $\mathrm{S}_{v}$ symbol.

The operator (4) describes the most general representation of the visual pattern, which corresponds to absence of limitations imposed on formation of the $G(v)$ set. It is equivalent to static realization of the IM.

\section{Features of the positional IM}

Our analysis of practical solutions for displays built on bar graph arrays showed that realization of the discrete-analogous data representation is determined by two main factors, namely: a visual form of information mapping and arrangement of mutual electric connections between display elements.

The analytical form of IM representation describes realization of $S_{v}$ symbols from $\alpha_{i}$ elements of the set $A$ as well as determines $i_{1}$ and $i_{2}$ meanings in (4). Widely spread is the positional IM, optical non-uniformity of which is formed from the only excited element of the display information area $[9,10]$. The weighting factor of this indication index coincides with the meaning of an imaged value. Therefore, for this sort of the positional IM, numbers of initial and final excited elements of indicator in the formed $\mathrm{S}_{v}$ symbol visual pattern coincide in (4), i.e. $i_{1}=i_{2}=v$. As a result, some dynamical realization is possible, but it loses its technical expediency, as the set $G(v)$ converts into the unity set.

Our comparison of reliability performances for the positional and bar graph IMs shows that the probability of developing situation when the operator does not get any information about the parameter controlled in the first case is much higher. A failure or degradation of even one IAE or one of the corresponding circuits in a display driver when using the positional IM consisting from one excited indication index results in full vanishing of display information. But in the same situation, using the bar graph IM, the operator obtains information about controlled parameter, although with some additive inaccuracy in the data mapped.

However, usage of the positional IM based on two adjacent excited IAE provides an opportunity to considerably increase the reliability of data representation. This approach enables to realize two IM versions. In the first one, excited in the scale is the element with the weighting factor that corresponds to the represented meaning as well as the element with that exceeding by unity the first meaning.

In the second case, data are represented by excited elements, the first of which has the weighting factor corresponding to the indicated value, and the second one that is less by unity than the main one. Realization of these IM versions can be represented in the following 


\section{A.V. Bushma et al.: Static realization of reliable positional indication}

forms stemming from (4):

$$
\begin{aligned}
& S_{v \Sigma} \Leftrightarrow G_{\Sigma}(v)=\bigcup_{i=v}^{v+1} a_{i} \\
& S_{v \Delta} \Leftrightarrow G_{\Delta}(v)=\bigcup_{i=v-1}^{v} a_{i}
\end{aligned}
$$

where $S_{v \Sigma}, S_{v \Delta}$ are visual symbols from alphabets for the two-element positional IM containing additive senior and junior indication indexes, respectively; $G_{\Sigma}(v), G_{\Delta}(v)$ are IAE sets $\alpha_{i}$ that are excited when forming, accordingly, the first and second IM versions.

Symbols of these IMs synthesized in accord with (5) and (6) create alphabets:

$$
\Omega_{\mathrm{IM} \Sigma}=\left\{S_{1 \Sigma}, S_{2 \Sigma}, \ldots, S_{v \Sigma}, \ldots, S_{(p-1) \Sigma}, S_{p \Sigma}\right\}
$$

$\Omega_{\mathrm{IM} \Delta}=\left\{S_{1 \Delta}, S_{2 \Delta}, \ldots, S_{v \Delta}, \ldots, S_{(p-1) \Delta}, S_{p \Delta}\right\}$

An analysis of (5) and (6) in combination with (3) shows that formation of such IMs needs display information areas consisting of $\alpha_{i}$-element sets having the following forms:

$$
\begin{aligned}
& A_{\Sigma}=\left\{a_{1}, a_{2}, \ldots, a_{i}, \ldots, a_{p}, a_{p+1}\right\} \\
& A_{\Delta}=\left\{a_{0}, a_{1}, \ldots, a_{i}, \ldots, a_{p-1}, a_{p}\right\}
\end{aligned}
$$

where $A_{\Sigma}, A_{\Delta}$ are IAE sets on which, respectively, the first and second versions of the considered IM are formed.

As seen from definitions (5) - (10) above, realization of the two-element positional IM with the alphabet length $s=p$ needs indicator with an information field consisting of $(p+1)$ elements. Their weighting factors change between 1 to $(p+1)$ for IM with an additive senior element and from 0 to $p$ for that with an additive junior element.

Juxtaposition of symbols from various IM versions by taking into account weighting factors of forming them IAE shows that they are identical except the most junior term $S_{1 \Delta}$ in one version and the most senior term $S_{p \Sigma}$ for another one, i.e., $S_{v \Sigma} \equiv S_{(v+1) \Delta}$ when $v=\overline{1,(p-1)}$. At the same time, considering an information aspect of data representation and taking into account (1) and (2), one can write the equality $I\left(S_{v \Sigma}\right) \equiv I\left(S_{v \Delta}\right)$. It is indicative of informational equivalency of symbols with identical positional numbers in these two versions of IM.

\section{Hardware realization of a highly reliable positional IM}

Let us consider some features of the device circuit technique that form suggested positional IM in a display with linear connections between IAE.

The most widely spread and practically applicable is the variant of bar graph array element connections with a common bus. To synthesize a visual symbol $S_{v}$ using this indicator, it is necessary to form a single-coordinate matrix (vector) of electric signals possessing the following appearance

$$
\mathbf{E}=\left\|e_{1}, e_{2}, \ldots, e_{i}, \ldots, e_{q}\right\|
$$

where $e_{i}$ is an excitation signal of the $i$-th IAE and $i=\overline{1, q}$.

In dependency on the used element type, either direct (alternating) voltage or current can serve as a signal. Such signal is generated with appropriate driver. Its functions comprises buffer transformation of logical signals from a control circuit. The number of driver channels is equal to the amount of display elements $q$. Absence of logical and mutual processing of control signals in channels determines identity of realized functions that can be described by the following operator

$$
e_{i}=f\left(z_{i}\right),
$$

where $f$ is a function describing transformation of control signals into the form corresponding to the IAE type; $z_{i}$ is the $i$-th output signal of a digital control circuit.

Usually, in currently optoelectronic informationmeasuring systems, displayed data are represented by the parallel binary code [9]. As a rule, it provides minimization of circuit solutions for processing signals due to excessless of such a form for digital data representation. To obtain display control signals in accord with (11), it is necessary to form a code with the number of digits equal to IAE amount. It is used to control the driver that realizes the condition (12). Consequently, the digital circuit for controlling the display consisting of $q$ IAEs fulfills logical processing signals of the following form

$$
Z=\psi_{z}(X) \text {, }
$$

where $Z$ is the $q$-digit output code, $Z=\left\{z_{1}, z_{2}, \ldots, z_{i}, \ldots, z_{q}\right\} ; \psi_{z}$ is the code translation operator; $X$ is the $k$-digit input code, $X=\left\{x_{1}, x_{2}, \ldots, x_{i}, \ldots, x_{k}\right\}$.

Let us assume the existence of a symbol reflecting zero value of an input signal and ascribe it the conventional positional number $v=1$. Then circuit realization of highly reliable positional IM, which correspond to operators (5) and (6), taking into account the way to obtain display excitation signals described by (11) and (12), can be written as follows:

$$
\begin{aligned}
S_{v \Sigma} \Leftrightarrow \mathbf{E}_{v \Sigma} & =\left\|0, \ldots, 0, f\left(z_{v}\right), f\left(z_{v+1}\right), 0, \ldots, 0\right\|= \\
& =\left\|e_{i}=f\left(z_{i \Sigma}\right)\right\|, \\
S_{v \Delta} \Leftrightarrow \mathbf{E}_{v \Delta} & =\left\|0, \ldots, 0, f\left(z_{v-1}\right), f\left(z_{v}\right), 0, \ldots, 0\right\|= \\
& =\left\|e_{i}=f\left(z_{i \Delta}\right)\right\|,
\end{aligned}
$$

where $\mathbf{E}_{v \Sigma}, \mathbf{E}_{v \Delta}$ are vectors of electrical signals providing formation of two-element positional IM with an additive senior and junior IAE, respectively; $z_{i \Sigma}, z_{i \Delta}$ correspond to the $i$-th code digit at the output of digital control circuit when synthesizing the IM with an addi- 


\section{A.V. Bushma et al.: Static realization of reliable positional indication}

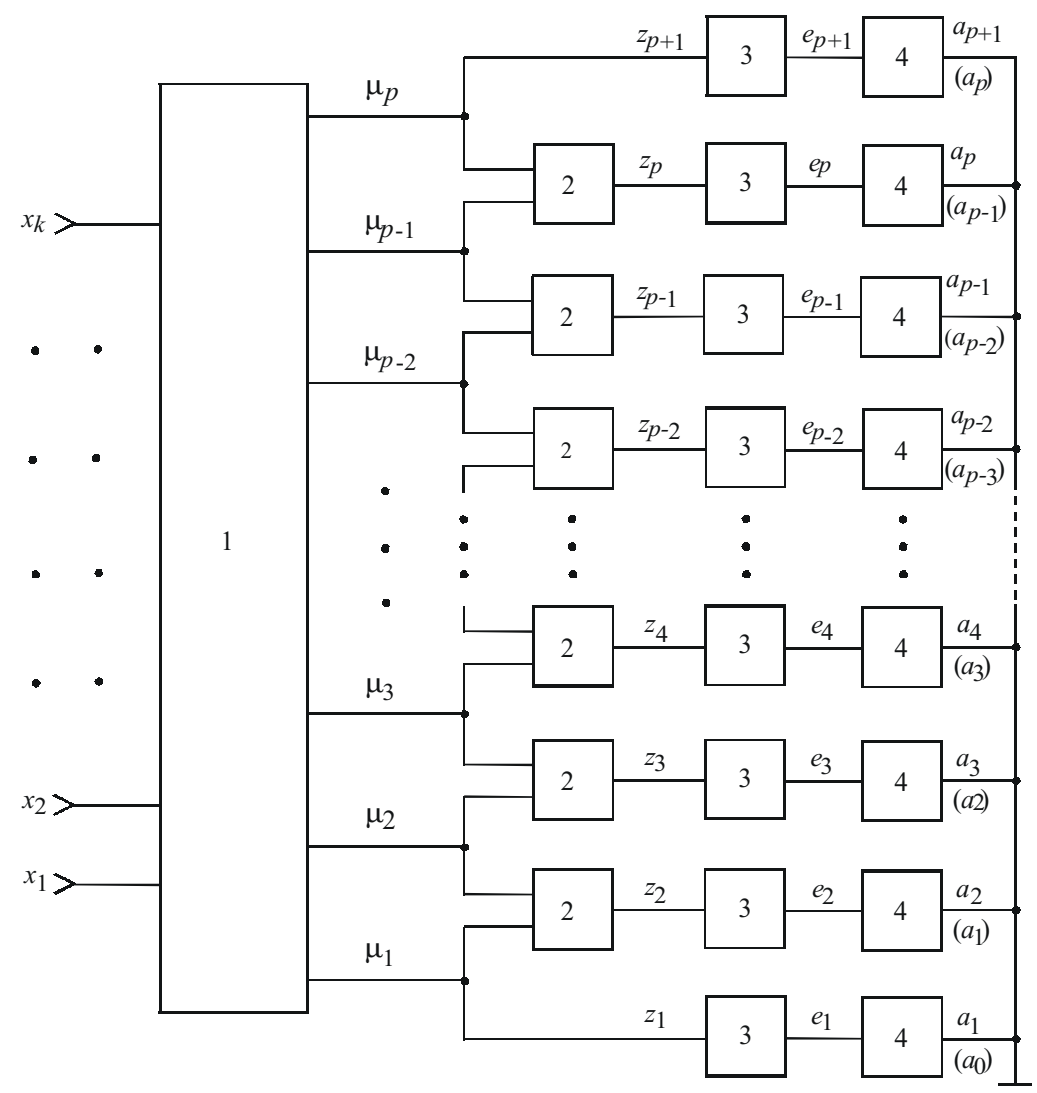

Fig. 1. The structural solution for reliable two-element positional indication, where 1 is decoder, 2 are OR elements, 3 are drivers and 4 are display elements.

tive senior and junior IAE, respectively.

Following (13), to excite display in accord with (14) and (15), digital control circuit should form the binary code $Z$. It is obvious that for considered variants of $S_{v}$ visual symbol formation, the transformation (13) with taken into account (14) and (15) can be converted into the following forms:

$$
\begin{aligned}
& S_{v \Sigma} \Leftrightarrow Z_{v \Sigma}=\left\{0, \ldots, 0, z_{v}, z_{v+1}, 0, \ldots, 0\right\}= \\
& =\psi_{z \Sigma}\left(X_{v}\right) \text {, } \\
& S_{v \Delta} \Leftrightarrow Z_{v \Delta}=\left\{0, \ldots, 0, z_{v-1}, z_{v}, 0, \ldots, 0\right\}= \\
& =\psi_{z \Delta}\left(X_{v}\right) \text {. }
\end{aligned}
$$

Let us develop some mathematical model of a digital structure that forms a two-element positional IM in the display consisting of $(p+1)$ IAEs with a common electrode. Control signals for the display are formed from the $k$-digit input code when implying $k=\mathrm{E}\left[\log _{2}(p-1)\right]+1$, where $\mathrm{E}=$ Entire. Then, stemming from (13) and taking into account (16) and (17), this structure can be described by the following models:

$$
\begin{aligned}
& z_{i \Sigma}=\mu_{i}+\mu_{i+1}, \\
& z_{i \Delta}=\mu_{i-1}+\mu_{i},
\end{aligned}
$$

where $\mathrm{m}_{i}$ is the $i$-th minterm of output signals, and $\mu_{0}=\mu_{p+1}=0$, as the input $k$-digit code has $p$ minterms.

The structural solution realized by the models (18) and (19) is depicted in Fig. 1. The decoder 1 forms $p$ minterms $\mu_{1} \ldots \mu_{p}$ from $k$ input variables $x_{1} \ldots x_{k}$. Obtained signals are processed by OR elements 2 . As $\mu_{p+1}=0$ for (18) and $\mu_{0}=0$ for (19), the amount of elements 2 is by unity less than the number of an input code minterms, i.e. it is equal to $(p-1)$. On their outputs, the code $Z_{v \Sigma}\left(Z_{v \Delta}\right)$ is formed. This code controls the array consisting from $(p+1)$ drivers 3 . Their output signals are connected with $(p+1)$ display elements $a_{i} 4$ that have one common output electrode.

They provide formation of $S_{v}$ symbol (in the kind of $S_{v \Sigma}$ or $S_{v \Delta}$ ), which is corresponded to the input code $X_{v}$. Our analysis of (7) - (10) as well as (18) - (19) showed that considered synthesis of $S_{v \Sigma}$ symbol on the set $A_{\Sigma}$ of IAE $a_{i}$ accordingly to (9), and $S_{v \Delta}$ symbol on $A_{\Delta}$ in accord with (10), from the practical viewpoint, differs by the measure that is considered here as a scale relatively to the display information area. Therefore, both models (18) and (19) are realized by the same digital structure. The difference consist in connection elements $a_{i} 4$ to the control circuit. In Fig. 1, designations of $a_{i}$ elements 4 from the set $A_{\Sigma}$ described by (9) are given without parentheses, and those from (10) are given in parentheses. 


\section{A.V. Bushma et al.: Static realization of reliable positional indication}

\section{Conclusions}

Thus, analyzed is the highly reliable positional information model and represented are logical operators that describe static formation of both its versions for displays with linear electrical connections between elements. We studied peculiarities of an arrangement of their information areas and alphabets of both information model versions. Offered and realized are mathematical models of respective circuit solutions for displays consisting from elements connected with a common electrode.

It is ascertained that the main properties of the elaborated information model and its hardware realization are as follows:

1) simplicity of visual symbol formation and used for that circuit solutions based on series IC regular structures;

2) realization of both IM versions by the same digital structure for any display consisting of elements with a common electrode;

3) to synthesize IM with an alphabet comprising $p$ symbols one needs the display with an information area consisting of $(p+1)$ elements.

The results obtained enable one to create highly reliable and effective display devices for optoelectronic information-measuring systems and to analytically describe the processes taking place in them. It gives designers of mass-produced devices and complex systems new means to analyze and separate the most effective structural and functional solutions providing a fast and reliable transfer of obtained data to an operator.

\section{References}

1. P.P. Ornatski, Theoretical principles of informationalmaesuring technique (in Russian). Kiev, Vyshcha shkola, $1983,455 \mathrm{p}$

2. V.I. Kostyuk, V.E. Khodakov, Systems for imaging information and engineer psychology (in Russian) 1977, 192 p.

3. N.A. Mitreykin, A.I. Ozerski, Reliability and testing radioparts and components (in Russian). Moscow, Radio i svyaz, 1981, $272 \mathrm{p}$.

4. B. S. Dhillon, Chanan Singh. Engineering Reliability New Techniques and Applications. A Wiley-Interscience. Publication John Wiley \& Sons. New York. - 1981.

5. Handbook: Engineer psychology (in Russian). Ed. by B.F. Lomov. Moscow, Mashinostroenie, 1982, 368 p.

6. Principles of engineer psychology (in Russian). Ed. by B.F. Lomov, Vysshaya shkola, 1977, 335 p.

7. F.M. Yablonski, Yu.V. Troitski. Means for imaging information (in Russian). Moscow, Vysshaya shkola, 1985, 200 p.

8. L.F. Kulikovski, V.V. Motov, Theoretical principles of informational processes (in Russian). Moscow, Vysshaya shkola, 1987, $248 \mathrm{p}$

9. Stan Gage, Mark Hodapp, Dave Evans, Hans Sorensen. Optoelectronics application manual. McGraw-Hill Book Company. New York. - 1977

10. V.A. Gorokhov, V.S. Rybakov, A.P. Shev"ev, Integrated indication appliences for control-measuring apparatus (in Russian) // Mikroelectronika i poluprovodnikovyye pribory. Moscow, Sov. radio, 1980, issue 5, p. $254-266$. 